\title{
Is nitric oxide the forgotten nephroprotective treatment during cardiac surgery?
}

\author{
Mina Khorashadi ${ }^{1}$, Michael P. Bokoch ${ }^{1}$ and Matthieu Legrand ${ }^{1,2^{*}}$ (])
}

Acute kidney injury (AKI) is a frequent complication in patients undergoing cardiac surgery with cardiopulmonary bypass $(\mathrm{CPB})$, with a reported incidence of $30-50 \%$, and an associated increase in mortality [1]. Proposed mechanisms of cardiac surgery-associated AKI include neurohormonal activation, inflammation, ischemiareperfusion injury, hypoperfusion, venous congestion, microembolization and hemolysis. Nitric oxide (NO) is an important homeostatic mediator of renal hemodynamics [2]. NO deficiency occurs in various experimental models of renal injury, including during $\mathrm{CPB}$. Data from animal studies suggest that abnormalities in endogenous NO production lead to kidney injury, due to an imbalance of production between the inducible and endothelial nitric oxide synthase [3].

The effect of NO therapy on AKI is controversial and may depend on the setting. In a randomized control trial including patients with multiple heart valve surgery $(n=244)$, NO delivered from the initiation of CPB reduced post-operative AKI and transition to stage 3 chronic kidney disease [4]. Contrary to these findings, a broadly inclusive meta-analysis (including studies of patients with sepsis and acute respiratory distress syndrome, ARDS, and surgical patients) showed no benefit of $\mathrm{NO}$ on post-operative renal function [5]. Of concern, the same study found that $\mathrm{NO}$ use was actually associated with worse renal function in patients with ARDS

*Correspondence: matthieu.legrand@ucsf.edu

${ }^{1}$ Department of Anesthesiology and Peri-Operative Medicine, University of California - UCSF Medical Center, 500 Parnassus Ave, San Francisco, CA 94143, USA

Full list of author information is available at the end of the article
[6]. Given these conflicting results, how should the benefit of NO for prevention of AKI in cardiac surgery be adjudicated?

The meta-analysis by $\mathrm{Hu}$ et al. in this issue of Annals of Intensive Care addresses several of these discrepancies [7]. The authors analyzed five randomized controlled trials that investigate the effects of perioperative NO therapy on AKI after cardiac surgery. Notably, the included studies differ in two major parameters: the timing of $\mathrm{NO}$ administration and the definition of AKI. Of those analyzed, the two older studies initiated NO therapy immediately before weaning from $\mathrm{CPB}$, and defined AKI as urine output less than $0.3 \mathrm{~mL} / \mathrm{kg} / \mathrm{h}$ or need for renal replacement therapy. These latter criteria likely underdiagnose the incidence of clinically significant AKI. In the three more recent studies (2018-2019), NO was initiated at the beginning of $\mathrm{CPB}$ and use the KDIGO criteria to define AKI. The pooled effect showed that perioperative NO therapy significantly reduced the risk of AKI after $\mathrm{CPB}$ with RR of 0.76 (95\% CI 0.62-0.93, $p=0.008$ ). Interestingly, NO therapy only showed benefit when initiated at the beginning of CPB (RR 0.71, 95\% CI 0.54-0.94, $p=0.02$ ). This result may imply that a key window exists where NO therapy can provide renal protection.

To interpret their findings, the authors emphasize the role of hemolysis in development of AKI after CPB (Fig. 1). Red blood cells are sheared as they go through the bypass circuit and release free hemoglobin $(\mathrm{Hb})$ into the circulation. Free $\mathrm{Hb}$ in turn scavenges NO produced by endothelial cells, depleting plasma levels and ultimately resulting in vasoconstriction and reduced organ perfusion. Furthermore, free $\mathrm{Hb}$ and its degradation byproducts cause glomerular injury by forming reactive oxygen species, which 


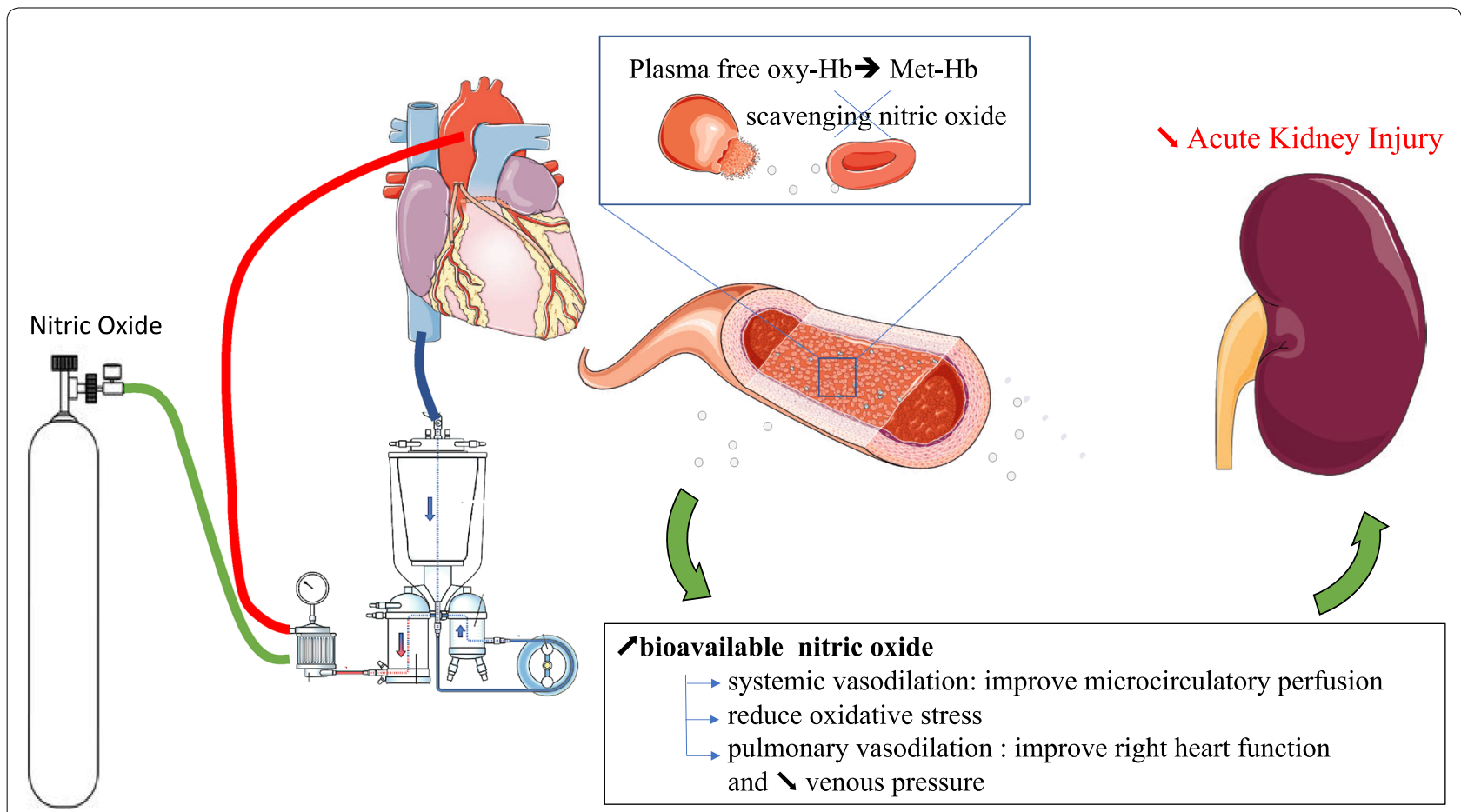

Fig. 1 Proposed mechanisms of renal protection using nitric oxide during cardiac surgery with cardio-pulmonary bypass. Free Oxy-Hb oxyhemoglobin, Met-Hb methemoglobin

can directly damage renal tubular cells and endothelium [3]. Thus, supplying exogenous NO in the peri-bypass period may not only protect organ perfusion by restoring the NO levels depleted by hemolysis, but also maybe by directly chelating free $\mathrm{Hb}$ and dampen its nephrotoxic effects. This is in contrast to ARDS where there is no apparent hemolysis. Moreover, prolonged NO therapy lasting days or even weeks (more commonly used in ARDS as compared with cardiac surgery) [6] has been associated with tubular apoptosis [8] and production of reactive nitrogen species leading to inflammation and renal injury.

The conclusions drawn by $\mathrm{Hu}$ et al. encounter several challenges [7]. First, the small number of studies included in the analysis yield low statistical power. To provide additional statistical context for their findings, the authors performed trial sequential analysis, a method for adjusting significance thresholds in meta-analyses when the optimal sample size has not been reached. Given that the TSA monitoring boundary was not exceeded, the authors duly maintain that further studies are needed to confirm their basic finding. Second, the effect of NO on right ventricular function and venous congestion, both strongly associated with renal dysfunction [9, 10], are not explored in this meta-analysis. Given that right heart dysfunction is common after $\mathrm{CPB}$, and that $\mathrm{NO}$ reduces right heart workload, the renal protective effects of $\mathrm{NO}$ may be mediated through improved right heart function. Unfortunately, of the studies included, only two reported hemodynamic data pertinent to right ventricular function, and the authors were unable to include this critical endpoint in their analysis. Finally, as discussed above, the finding that NO therapy lacks benefit when initiated only at the end of CPB is strongly confounded by the various definition of AKI used and the mode of administration of NO (i.e. inhaled or through the CPB circuit). This analysis is nevertheless important because it suggests that better delineation of the timing of NO therapy and its efficacy for preventing AKI warrants further investigation.

Limitations aside, what can clinicians learn from this information to help care for patients undergoing cardiac surgery? While no published trial to date has reported any adverse effects of $\mathrm{NO}$ during $\mathrm{CPB}$, given the costs associated with $\mathrm{NO}$ therapy, its routine implementation for the sole indication of protecting the kidney is not practical in the absence of further data. The findings by $\mathrm{Hu}$ et al. may be limited by the small, heterogeneous pool of available literature, but they inform important new hypotheses about the timing and mechanisms of peribypass NO therapy. New studies inspired by this work are badly needed to better understand and manage AKI in this vulnerable patient population. 


\section{Authors' contributions}

MK, MB and ML drafted the manuscript. All authors read and approved the final manuscript.

\section{Funding}

No funding for this publication.

\section{Availability of data and materials}

Not applicable.

\section{Ethics approval and consent to participate}

Not applicable.

\section{Consent for publication}

Not applicable.

\section{Competing interests}

$M L$ received research funding from the French ministry of health, research support from Sphingotec, lecture fees from Fresenius and Baxter and consulting fees from Novartis. The other authors declare that they have no competing of interests.

\section{Author details}

${ }^{1}$ Department of Anesthesiology and Peri-Operative Medicine, University of California - UCSF Medical Center, 500 Parnassus Ave, San Francisco, CA 94143, USA. ${ }^{2}$ Institute National de la Santé et de la Recherche Médicale (INSERM) 942, Lariboisière Hospital \& F-CRIN INI-CRCT, Paris, France.

Received: 12 December 2019 Accepted: 23 January 2020

Published online: 12 February 2020

\section{References}

1. Thiele RH, Isbell JM, Rosner MH. AKI associated with cardiac surgery. Clin J Am Soc Nephrol. 2015;10:500-14.
2. Vermeulen Windsant IC, de Wit NCJ, Sertorio JTC, van Bijnen AA, Ganushchak YM, Heijmans JH, et al. Hemolysis during cardiac surgery is associated with increased intravascular nitric oxide consumption and perioperative kidney and intestinal tissue damage. Front Physiol. 2014:5:340.

3. Legrand M, Mik EG, Johannes T, Payen D, Ince C. Renal hypoxia and dysoxia after reperfusion of the ischemic kidney. Mol Med. 2008;14:502-16.

4. Lei C, Berra L, Rezoagli E, Yu B, Dong H, Yu S, et al. Nitric oxide decreases acute kidney injury and stage 3 chronic kidney disease after cardiac surgery. Am J Respir Crit Care Med. 2018;198:1279-87.

5. Ruan S-Y, Huang T-M, Wu H-Y, Wu H-D, Yu C-J, Lai M-S. Inhaled nitric oxide therapy and risk of renal dysfunction: a systematic review and metaanalysis of randomized trials. Crit Care. 2015;19:137.

6. Gebistorf F, Karam O, Wetterslev J, Afshari A. Inhaled nitric oxide for acute respiratory distress syndrome (ARDS) in children and adults. Cochrane Database Syst Rev. 2016. https://doi.org/10.1002/14651858.CD002787. pub3.

7. Hu J, Spina S, Zadek F, Kamenshchikov NO, Bittner EA, Pedemonte J, et al. Effect of nitric oxide on postoperative acute kidney injury in patients who underwent cardiopulmonary bypass: a systematic review and metaanalysis with trial sequential analysis. Mais Ann Intensive Care. 2019;9:129.

8. Goździk W, Albert J, Harbut P, Zieliński S, Ryniak S, Lindwall R, et al. Prolonged exposure to inhaled nitric oxide transiently modifies tubular function in healthy piglets and promotes tubular apoptosis. Acta Physiol. 2009; 195:495-502.

9. Legrand M, Mebazaa A, Ronco C, Januzzi JL. When cardiac failure, kidney dysfunction, and kidney injury intersect in acute conditions: the case of cardiorenal syndrome. Crit Care Med. 2014;42:2109-17.

10. Coutrot M, Dépret F, Legrand M. Is nitric oxide nephro- or cardioprotective? Am J Respir Crit Care Med. 2019;199:1441-2.

\section{Publisher's Note}

Springer Nature remains neutral with regard to jurisdictional claims in published maps and institutional affiliations.

\section{Submit your manuscript to a SpringerOpen ${ }^{\circ}$ journal and benefit from:}

- Convenient online submission

- Rigorous peer review

- Open access: articles freely available online

- High visibility within the field

- Retaining the copyright to your article

Submit your next manuscript at $\boldsymbol{\nabla}$ springeropen.com 